\title{
Overview of the ECE measurements on EAST
}

Yong Liu ${ }^{1, *}$, Hailin Zhao ${ }^{1}$, Tianfu Zhou ${ }^{1}$, Xiang Liu ${ }^{1}$, Zeying Zhu ${ }^{1}$, Xiang Han ${ }^{1}$, Stefan Schmuck ${ }^{2}$, John Fessey ${ }^{2}$, Paul Trimble $^{2}$, C.W. Domier ${ }^{3}$, N.C. Luhmann, Jr. ${ }^{3}$, Ang $\mathrm{Ti}^{1}$, Erzhong $\mathrm{Li}^{1}$, Bili Ling ${ }^{1}$, Liqun $\mathrm{Hu}^{1}$, Xi Feng ${ }^{4}$, Ahdi Liu ${ }^{4}$, W.L. Rowan $^{5}$, He Huang ${ }^{5}$, P.E. Phillipss ${ }^{5}$, and Lorenzo Figini ${ }^{6}$

${ }^{1}$ Institute of Plasma Physics, Chinese Academy of Sciences, Hefei 230031, China

${ }^{2}$ CCFE, Culham Science Centre, Abingdon, Oxon, OX14 3DB, UK

${ }^{3}$ Department of Applied Science, University of California at Davis, Davis, California 95616, USA

${ }^{4}$ University of Science and Technology of China, Hefei 230026, China

${ }^{5}$ Institute for Fusion Studies, University of Texas at Austin, TX 78712, USA

${ }^{6} \mathrm{CNR}$, Istituto di Fisica del Plasma, Milano 20125, Italy

\begin{abstract}
Radiometer systems and a Michelson interferometer, have been operated routinely to detect the electron cyclotron emission (ECE) from EAST plasmas for diagnosing the local electron temperature. A common quasi-optical antenna placed inside the vacuum vessel is employed to collect and focus the plasma emission, and the line of sight is along a radial chord. All of the systems are located in a diagnostic room where the plasma emission is transmitted by overmoded corrugated waveguide. In-situ absolute intensity calibration has been carried out for both the radiometer systems and the Michelson interferometer independently, to ensure that the ECE diagnostic provides an independent electron temperature measurement. In order to diagnose the small-amplitude electron temperature fluctuation, a correlation ECE (CECE) diagnostic has been designed and commissioned recently. The CECE diagnostic employs an independent antenna system which has improved poloidal resolution. A synthetic diagnostic is realized by using the simulation code SPECE to interpret the ECE data in plasmas with non-Maxwellian distribution, and preliminary results imply that the ECE data could be still useful as a localized measurement in plasmas with non-thermal electrons, such as the LHW-heated plasmas on EAST.
\end{abstract}

\section{Introduction}

Electron cyclotron emission (ECE) measurements have been widely employed in tokamak plasmas since the 1970s as a powerful diagnostic [1, 2]. Generally, ECE provides the temporal evolution of the local electron temperature with good spatial and temporal resolutions. In addition, information about the electron density, the electron velocity distribution, and the wall reflectivity can also be inferred from the entire ECE spectrum [3, 4].

On the experimental advanced superconducting tokamak (EAST), the ECE diagnostic has been operated since the first plasma of EAST and was improved continuously since then. There are a few radiometer systems $[5,6]$ and a Michelson interferometer [7] for conventional ECE measurement to diagnose the electron temperature profiles and electron temperature fluctuation driven by macroscopic instabilities. A correlation ECE (CECE) diagnostic has been developed to detect the turbulence associated smallamplitude electron temperature fluctuation. Synthetic diagnostic is studied by using the forward modeling code SPECE, and this facilitates the explanation of ECE data in plasmas with non-Maxwellian distribution. The conventional ECE diagnostics on EAST will be described in Section 2, and Section 3 introduces the CECE diagnostic.

*e-mail: liuyong@ipp.ac.cn
Results of synthetic diagnostic are given in Section 4. Section 5 is the summary.

\section{Conventional ECE diagnostic}

\subsection{Antenna and transmission line}

Since 2014, a quasi-optical (QO) antenna has been adopted for collecting and focusing EAST plasma emission along a horizontal line of sight. As shown in Figure 1, the antenna comprises 3 reflectors: an ellipsoidal mirror and two flat mirrors. The ellipsoidal mirror can be rotated facing either the plasma or a hot-load calibration source. The ellipsoidal mirror is located $2000 \mathrm{~mm}$ away from the plasma center, and the distance to the waveguide is 1000 $\mathrm{mm}$. The ellipsoidal mirror has an effective focal length of $900 \mathrm{~mm}$, and forms a beam size of roughly $40 \mathrm{~mm}$ for a toroidal magnetic field of $2.3 \mathrm{~T}$.

A single flat window with thickness of $15 \mathrm{~mm}$ was used in the year 2014, and this results in parallel-plate interference effects and consequently transmission ripple. Even though the in-situ absolute intensity calibration has been carried out, the transmission ripple has influence on the measurement and it is difficult to evaluate this effect quantitatively. In order to reduce the transmission ripple, the flat window was replaced with a double wedge window 


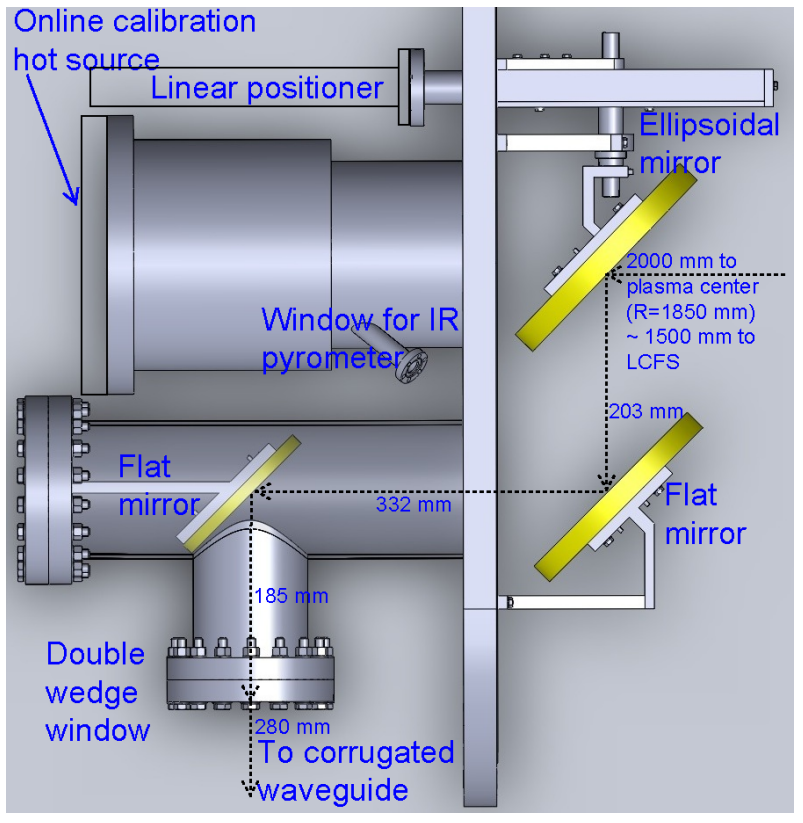

Figure 1. Quasi-optical antenna for the ECE diagnostic on EAST.

in 2015. Figure 2 shows the simulation results of the transmission for the single flat window and the double wedge window. The parameters of the double wedge window are: the wedge angle $\Psi$ is 12.5 degree, the thickness of the thin side $d_{1}$ is $10 \mathrm{~mm}$, and the distance between both windows $d_{w}$ is $1 \mathrm{~mm}$. The material for both the flat window and the double wedge window is high density polyethylene with dielectric constant $\epsilon_{r}$ of 2.3.

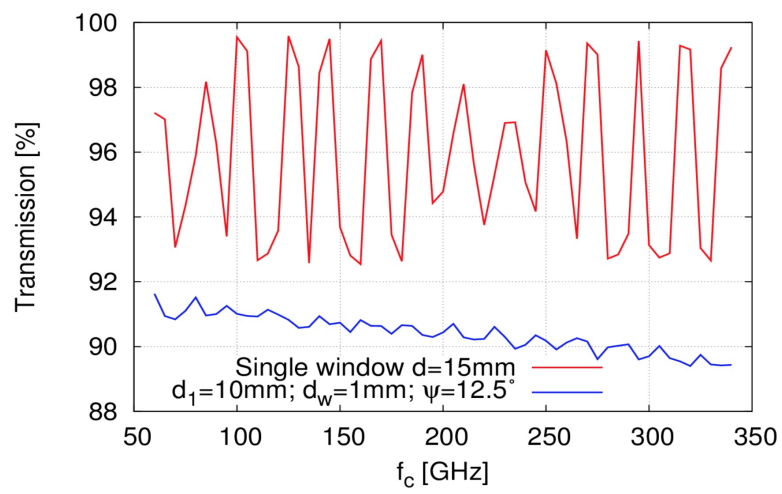

Figure 2. Comparison of the transmission for a single flat window and a double wedge window.

A wire grid polarizer is placed between the window and the waveguide to select the extraordinary mode (Xmode) polarization. Low-loss corrugated waveguides with $63.5 \mathrm{~mm}$-id is used. The transmission line (TL) comprises roughly 45 meters of waveguides, and 8 miter bends. Because some bends are not oriented with identical or orthogonal planes of incidence, the linear polarization is rotated by roughly 63.15 degrees after transmission through the 8 miter bends.
In the diagnostic room, a notch filter is used to prevent the measurement systems from being damaged by electron cyclotron heating $(\mathrm{ECH})$ stray radiation and then the plasma emission is divided into 3 paths by using two wire grid polarizers (see Figure 3 ). The power ratio for the three paths is 5:4:1. The first two paths are for the radiometer systems, and the remaining one is for the Michelson interferometer.

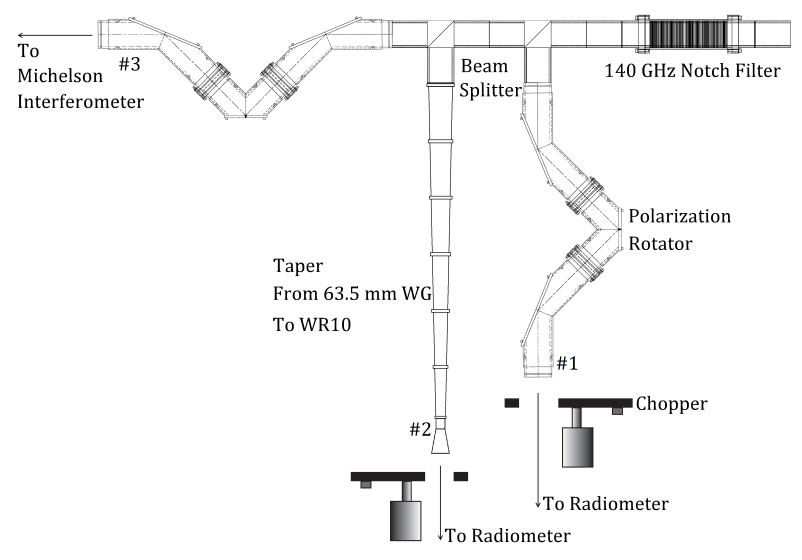

Figure 3. The layout of the transmission line (TL) in the diagnostic room.

\subsection{ECE measurement systems}

\subsubsection{Radiometer systems}

A 16-channel radiometer system which has a frequency coverage of $98.5-126 \mathrm{GHz}$ has been operated since the first plasma of EAST in 2006 [5]. In 2012, a 32-channel radiometer system was designed and commissioned to measure the plasma emission in the frequency range of 104$168 \mathrm{GHz}$ [6], and it uses the \#1 output of the TL. This 32-channel radiometer has been upgraded in 2015, and the frequency interval for the lower frequency range upto 120 $\mathrm{GHz}$ is reduced to $1 \mathrm{GHz}$ from $2 \mathrm{GHz}$ by introducing 8 channels in the intermediate frequency (IF) part. In order to extend the frequency coverage, an independent 8channel radiometer system covering a frequency range of 97-104 GHz (local oscillator of frequency $90 \mathrm{GHz}$ ) was built in 2015, and it uses the \#2 output of the TL. With the combination of these radiometer systems, they have a frequency coverage capable of measuring the second harmonic ECE for plasmas with toroidal $B_{t}$ higher than 1.75 $\mathrm{T}$.

The new system has been fully characterized in the laboratory, and the test focuses on the performance of the IF bandwidth and the linearity. The results of the linearity test (see Figure 4 (a) and 9c)) indicate a good linearity in the range from 0 to $2 \mathrm{~V}$. The sensitivity of each channel (including the RF part) has been adjusted to ensure that the system output in plasma measurements is within this dynamic range. The frequency response of the heterodyne radiometer electronics is shown in Figure 4 (b) and (d), and the result shows that each channel is responsive to only a single band of radio frequency (RF) frequencies. 
The RF bandwidth $f_{R F}$ of the radiometer systems varies from roughly $250 \mathrm{MHz}$ to $500 \mathrm{MHz}$, and the video bandwidth $f_{V}$ of the systems is upto $400 \mathrm{kHz}$. This results in a sensitivity of $4-5.7 \%\left(\approx \sqrt{\frac{2 f_{V}}{f_{R F}}}\right)$. This is adequate for most applications of ECE measurements such as diagnosing the electron temperature profile and the electron temperature fluctuation driven by MHD instabilities. However, it is not adequate to detect the small-amplitude, highfrequency fluctuations (with typical amplitudes around $1 \%$ ) driven by micro-instabilities.
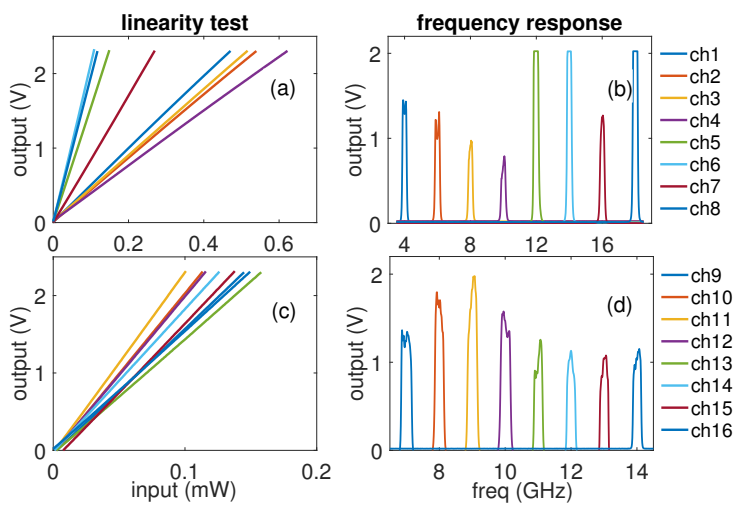

Figure 4. Tests of IF linearity ((a) and (c)) and frequency response ((b) and (d)) for the new 16 channels. The IF central frequency of the top is from 4 to $18 \mathrm{GHz}$, with $2 \mathrm{GHz}$ separation, while the bottom is from 7 to $14 \mathrm{GHz}$, with $1 \mathrm{GHz}$ separation.

\subsubsection{Michelson interferometer}

One of JET's Michelson interferometers was loaned to EAST in 2012, and it has been operated routinely sine the year 2014 [7]. This Michelson interferometer is one of the original systems developed by SPECAC in the 1980s for the ECE measurements in magnetically confined plasmas. This system had not been in operation since the late 1990s, and it has been refurbished when the loan agreement was signed in late 2011.

The system has a spectral resoluton of $2.7 \mathrm{GHz}$, and a temporal resolution of roughly $33 \mathrm{~ms}$. The instrument is able to measure the ECE spectra from $80 \mathrm{GHz}$ to 350 $\mathrm{GHz}$, and this is mainly limited by the transmission line and the in-situ calibration. This frequency range typically covers the first to fourth ECE harmonic ranges for EAST magnetic field configuration.

\subsubsection{In-situ intensity calibration}

In-situ absolute intensity calibration has been carried out independently for the radiometer systems and the Michelson interferometer since 2014 [8]. Two types of hot sources have been developed for the calibration on EAST. One of them (\#1) is a copy of the JET hot source [9], and the other one (\#2) is the ITER prototype developed by the Institute for Fusion Studies [10]. Source \#1 operates at atmosphere, while source \#2 needs to be operated in vacuum. Normally, an in-situ calibration was carried out before an experimental campaign starts by using the hot source \#1.

A digital coherent averaging technique and lock-in amplifier are separately used to measure the weak signal (a few tens $\mu \mathrm{V}$ ), and both of the methods require the input signal to be modulated by an optical chopper. The lockin amplifier gives RMS (root mean square) value of the modulated signal, while the coherent averaging technique results in the peak-to-peak value. The coherent averaging technique is more efficient because one can analyze all channels simultaneously.

Following the in-situ intensity calibration, the ECE diagnostic on EAST provides independent measurements of the electron temperature profiles and electron temperature fluctuation. Figure 5 shows the measurement of a few electron temperature profiles. The left figure of Figure 5 is for a discharge at a toroidal magnetic field of $1.8 \mathrm{~T}$. The black line is the electron temperature measured by a Michelson interferometer [7], which can give the profile of the whole plasma region with a temporal resolution of roughly 33 $\mathrm{ms}$, the red closed dots are the electron temperature measured by the existing 32-channel heterodyne radiometer, and the blue closed dots are the electron temperature measured by the newly upgraded 16-channel heterodyne radiometer. We can see that at such low magnetic field, the upgraded heterodyne radiometer can give the high field side electron temperature profile. The right figure of Figure 5 is the electron temperature profile for a discharge at a toroidal magnetic field of $2.47 \mathrm{~T}$, and the result demonstrates that the radial separation is decreased with the new 48 -channel heterodyne radiometer, from $\sim 4 \mathrm{~cm}$ to $\sim 2 \mathrm{~cm}$.
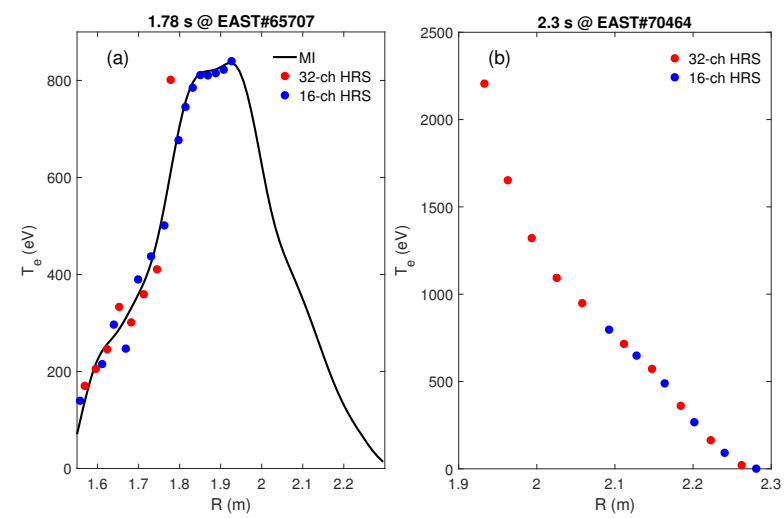

Figure 5. The electron temperature profiles at toroidal magnetic field of $1.80 \mathrm{~T}$ (left) and $2.47 \mathrm{~T}$ (right).

\section{Correlation ECE diagnostic}

In order to detect the small-amplitude electron temperature fluctuation associated with the anomalous transport, a CECE diagnostic has been designed since 2016. An independent QO antenna was designed, which has an improved poloidal resolution (roughly $15 \mathrm{~mm}$ for the frequency range of the CECE diagnostic). This is essential for the CECE diagnostic, and it determines the maximum 
wavenumber of the detected fluctuation. As illustrated in Figure 6, the QO antenna comprises a flat mirror and an ellipsoidal mirror. The ellipsoidal mirror is located 800 $\mathrm{mm}$ away from the plasma center, and the distance to the waveguide is $616 \mathrm{~mm}$. The ellipsoidal mirror has an effective focal length of $390.6 \mathrm{~mm}$. This antenna is integrated for both the CECE diagnostic and an existing Doppler backscatter diagnostic, and the flat mirror is rotatable in the poloidal direction. In contrast to the conventional ECE diagnostic, the heterodyne radiometer part of the CECE diagnostic is placed a few meters away from the diagnostic port.

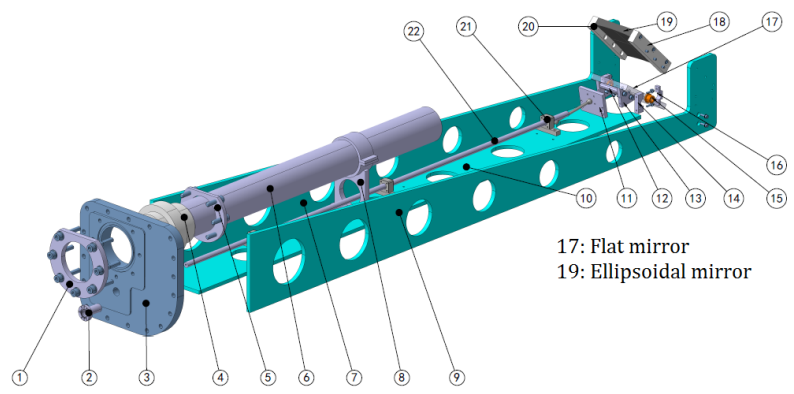

Figure 6. QO antenna of the CECE diagnostic.

The heterodyne detection part (see Figure 7) is similar to a conventional radiometer system, and a unique aspect is the application of YIG filters. The YIG filters (OMNIYIG INC) are of tunable central frequency in the frequency range of 4-18 GHz, and a $3 \mathrm{~dB}$ bandwidth of 100$250 \mathrm{MHz}$ (central frequency dependent). This feature is very important for facilitating the radial correlation. The sensitivity of this CECE diagnostic $\left(\sqrt{\frac{1}{\sqrt{N}} \frac{2 f_{V}}{f_{R F}}}\right.$, where $N$ is the number of data points for correlation) can reach around $0.2-0.3 \%$ for a correlation analysis using $10^{6}$ data points.

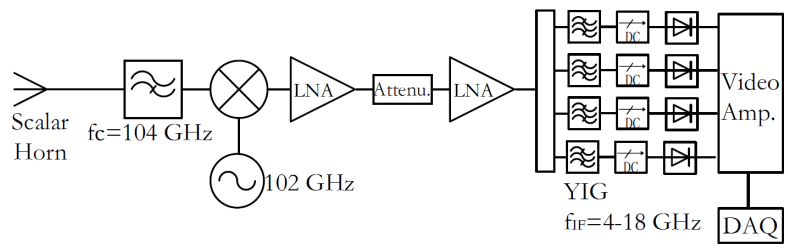

Figure 7. The heterodyne detection part of the CECE diagnostic.

This system has been commissioned in the EAST experimental campaign carried out in 2017. Preliminary results indicate that the broadband electron temperature fluctuations are detected in some cases. More tests will be conducted to confirm the observations in future.

\section{Synthetic diagnostic}

In order to interpret the ECE data in plasmas with nonMaxwellian distribution, the ECE ray-tracing code SPECE [11], developed as a support tool for the oblique ECE diagnostic at JET, is adopted for simulating the ECE spectrum in terms of radiative temperature on EAST. The input data for SPECE is described as follows: (i) the magnetic equilibrium is from EFIT, (ii) the electron temperature profile and the electron density profile are respectively provided by the Thomson scattering diagnostic and the reflectometry system, (iii) the LOS is described based on the real ECE diagnostic configuration. With the input data, the code solves the radiation transport equation along the ray trajectories in a tokamak plasma. Ray trajectories are computed making use of the cold dispersion relation, while the absorption and emission coefficients are obtained solving the relevant fully relativistic dispersion relation valid at high electron temperature. The actual antenna pattern is simulated by means of a multi-rays calculation, and the spatial resolution of the ECE measurements is computed by means of an algorithm that takes properly into account the emission along each ray of the beam.
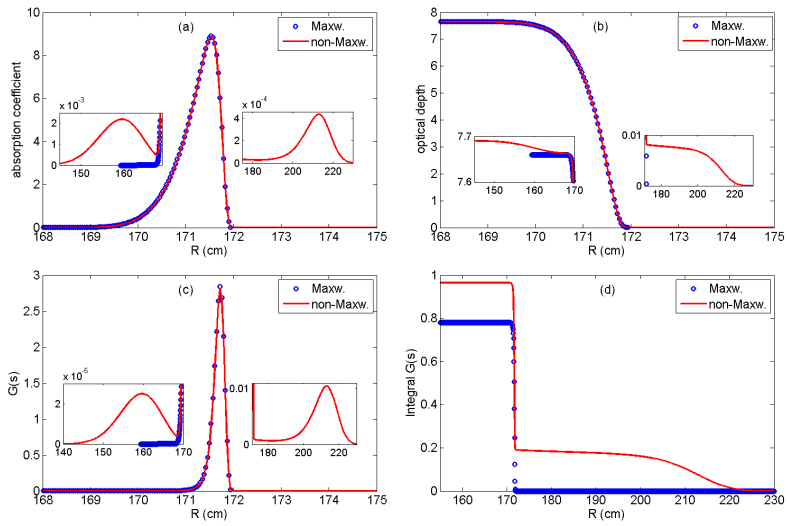

Figure 8. Example illustrating the spatial localization of 110 $\mathrm{GHz}$ ECE for plasmas $\left(\mathrm{B}_{0}=1.8 \mathrm{~T} @ 1.85 \mathrm{~m}\right)$ separately with Maxwellian and non-Maxwellian distribution functions: (a) absorption coefficient $\alpha(s)$, (b) optical depth $\tau(s)$, (c) $G(s)=$ $\frac{\omega^{2}}{8 \pi^{3} c^{2}} T_{\text {rad }}(s) \alpha(s) e^{-\tau(s)}$, (d) $\int G(s) d s$. Here, $s$ is along the major radius.

Figure 8 illustrates the spatial localization of $110 \mathrm{GHz}$ (corresponds to the second harmonic ECE for the case with Maxwellian distribution) for plasmas $\left(\mathrm{B}_{0}=1.8 \mathrm{~T} @\right.$ $1.85 \mathrm{~m}$ ) separately with Maxwellian and non-Maxwellian distribution functions. As the results (see Figure 8 (a)) show, two additional resonance layers will appear in separately HFS and LFS for the case with non-thermal electrons. The resonance layer in HFS is due to the relativistic down-shift of the second harmonic, while the resonance layer in the LFS is due to the relativistic down-shift of the third harmonic. Propagating from HFS to LFS, the emission in HFS will be absorbed completely for frequencies with high optical depth, and eventually the received intensity from LFS (the line of sight for a conventional ECE diagnostic) is mainly attributed to two emission layers (see Figure 8 (c)). In the rest of this paper, these two emission layers are named as the thermal emission layer and the non-thermal emission layer. As indicated in Figure 8 (c), the thermal emission layer for the non-Maxwellian case is close to that for the Maxwellian case. The reason is that the optical depth of the non-thermal emission is small 
(as shown in the small panel at the right side of the Figure 8 (b)), and consequently the absorption of the thermal emission layer is negligible when the emission propagates through the non-thermal resonance layer. Even though the non-thermal emission layer is much broader than the thermal emission layer, the intensity is much weaker.

In order to study the influence of the non-thermal electrons on the spatial localization of ECE measurement, a parameter $R=\int G_{\text {thermal }}(s) d s / \int G(s) d s$ is defined to describe the contribution of the thermal emission layer (local electron temperature) to the received intensity by an actual ECE diagnostic located in LFS, where the numerator is the integral for the thermal emission layer. The other parameter is the localization of the non-thermal emission layer, and it is described by the position of the peak value and the FWHM of the non-thermal emission layer.

To qualitatively study the contribution of the thermal emission layer to the total intensity and the localization of the non-thermal emission layer, some of the parameters describing the non-thermal electrons are scanned. The simulation results are summarized in Figures 9 and 10 . The input data such as the electron temperature profile, the electron density profile, and the magnetic equilibrium for the simulations are from measurements. The main parameters are: central electron density $n_{e 0}=3.5 \times 10^{19} \mathrm{~m}^{-3}$, central electron temperature $T_{e 0}=1100 \mathrm{eV}$, central magnetic field strength $B_{0}=1.8 \mathrm{~T}$, the major radius $R_{0} \approx$ $1.85 \mathrm{~m}$, the minor radius $a \approx 0.45 \mathrm{~m}$.

As illustrated in Figure 8 (c), for the frequency range with associated high optical depth and the non-thermal resonance layer at the HFS is behind the thermal resonance layer, the emission is absorbed completely and will not be detected at the LFS. This corresponds to radial range with major radius of roughly 1.6-2.1 m in Figures 9 and 10. Because the non-thermal emission layer is attributed to the third harmonic emission, it is obvious that the influence is stronger at the HFS and the influenced raidal range goes to LFS deeper as the energy of the non-thermal electrons increase (Figures 9 (d)). For a set of reasonable parameters $\left(\eta_{0}=0.001-0.002, \psi_{0}=0.4, \Delta \psi=0.3\right.$, $T_{\text {tail }}=25-30 \mathrm{keV}$, these parameters give a good agreement between the simulated and the experimental spectra [12]), the intensity attributed to the thermal emission layer is larger than $70 \%$ for the radial range $1.7-2.1 \mathrm{~m}$. For the radial range with major radius larger than $2.1 \mathrm{~m}$, the optical depth becomes smaller. Hence, the emission from the non-thermal resonance layer at the HFS is not absorbed completely and contributes to the observed intensity. This explains why there is a drop for most cases in Figure 9. As the location of the non-thermal electrons $\left(\psi_{0}\right)$ changes from plasma core to plasma edge, the number of resonant electrons becomes smaller and the contribution of the nonthermal electrons is smaller (see Figure 9 (b)).

As Figure 10 shows, the localization of the nonthermal emission layer (position and width) is strongly dependent of the radial position and radial distribution of the non-thermal electrons, and the dependence on the fractional density and tail energy is weak.
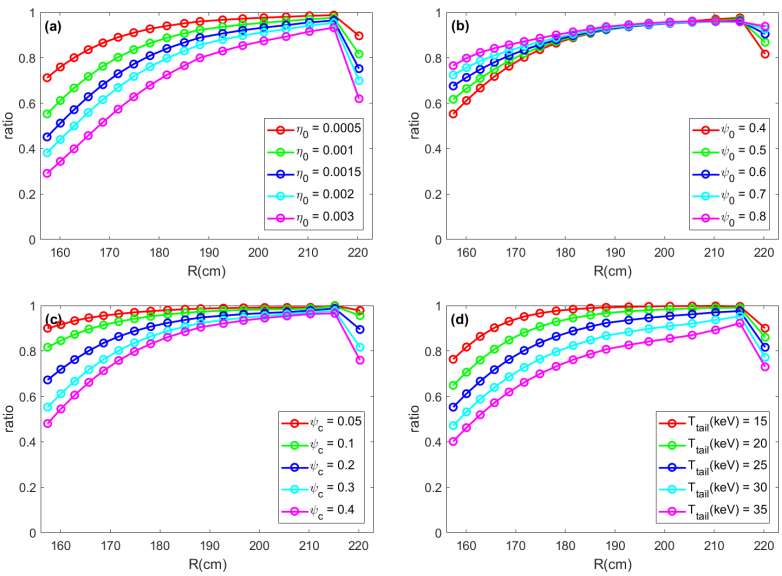

Figure 9. The ratio between intensity attributed to the thermal emission layer and the total intensity for scan of (a) the fractional density $\left(\psi_{0}=0.4, \Delta \psi=0.3, T_{\text {tail }}=25 \mathrm{keV}, u_{\|, \max }=0.4\right)$, (b) the radial position $\left(\eta_{0}=0.001, \Delta \psi=0.3, T_{\text {tail }}=25 \mathrm{keV}\right.$, $\left.u_{\|, \max }=0.4\right)$, (c) the radial distribution $\left(\eta_{0}=0.001, \psi_{0}=0.4\right.$, $\left.T_{\text {tail }}=25 \mathrm{keV}, u_{\|, \max }=0.4\right)$, and (d) the tail energy $\left(\eta_{0}=0.001\right.$, $\left.\psi_{0}=0.4, \Delta \psi=0.3, u_{\|, \max }=0.4\right)$.
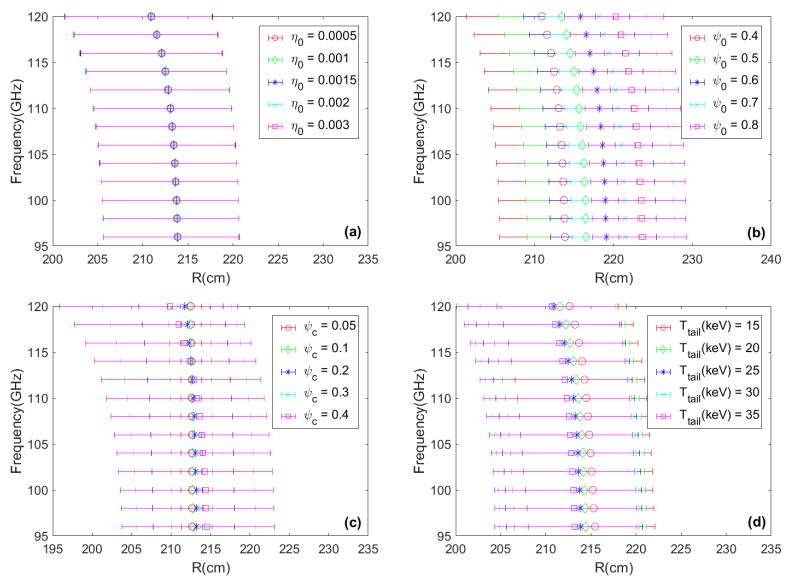

Figure 10. The localization (position defined at the peak value, width defined as the FWHM) of the non-thermal emission layer for scan of (a) the fractional density $\left(\psi_{0}=0.4, \Delta \psi=0.3, T_{\text {tail }}=\right.$ $\left.25 \mathrm{keV}, u_{\|, \max }=0.4\right)$, (b) the radial position $\left(\eta_{0}=0.001, \Delta \psi=\right.$ $\left.0.3, T_{\text {tail }}=25 \mathrm{keV}, u_{\|, \max }=0.4\right)$, (c) the radial distribution $\left(\eta_{0}=\right.$ $\left.0.001, \psi_{0}=0.4, T_{\text {tail }}=25 \mathrm{keV}, u_{\|, \max }=0.4\right)$, and (d) the tail energy $\left(\eta_{0}=0.001, \psi_{0}=0.4, \Delta \psi=0.3, u_{\|, \max }=0.4\right)$.

\section{Summary}

A complete set of measurement systems, including a number of radiometer systems and a Michelson interferometer, have been developed and operated on EAST to detect the plasma emission in the ECE frequency range. Two independent QO antennas were adopted to collect the plasma emission for the conventional ECE diagnostic and the CECE diagnostic. In-situ intensity calibration has been carried out to ensure independent electron temperature measurement since 2014. Following the calibration, the ECE diagnostic on EAST provides the information of electron temperature profiles and fluctuation with 
fairly good spatial and temporal resolutions. Synthetic diagnostic using forward modeling is used to interpret the ECE data in plasmas with non-Maxwellian distribution, and preliminary results imply that the ECE data could be still useful as a localized measurement in plasmas with non-thermal electrons, such as the LHW-heated plasmas on EAST.

\section{Acknowledgements}

This work was supported by the National Natural Science Foundation of China under Grant No. 11405211, and the Innovative Program of Development Foundation of Hefei Center for Physical Science and Technology. This work was also partly supported by National Magnetic Confinement Fusion Science Program of China under Contract Nos. 2015GB101003 and 2015GB103002.

\section{References}

[1] A.E. Costley, TFR Group, Phy. Rev. Lett. 38, 1477 (1977)

[2] I.H. Hutchinson, D.D. Komm, Nuclear Fusion 17, 1077 (1977)

[3] L. Figini, S. Garavaglia, E. De La Luna, D. Farina, P. Platania, A. Simonetto, C. Sozzi, JET-EFDA Contributors, Rev. Sci. Instrum. 81, 10D937 (2010)
[4] S. Schmuck, J. Fessey, J.E. Boom, L. Meneses, P. Abreu, E. Belonohy, I. Lupelli, Rev. Sci. Instrum. 87, 093506 (2016)

[5] A. Ti, B.L. Ling, Q.S. Fei, X. Gao, Int. J. Infrared Milli. Waves 28, 243 (2007)

[6] X. Han, X. Liu, Y. Liu, C.W. Domier, N.C. Luhmann, Jr., E.Z. Li, L.Q. Hu, X. Gao, Rev. Sci. Instrum. 85, 073506 (2014)

[7] Y. Liu, S. Schmuck, H.L. Zhao, J. Fessey, P. Trimble, X. Liu, Z.Y. Zhu, Q. Zang, L.Q. Hu, Plasma Science and Technology 18, 1148 (2016)

[8] X. Liu, H.L. Zhao, Y. Liu, E.Z. Li, X. Han, C.W. Domier, N.C. Luhmann, Jr., A. Ti, L.Q. Hu, X.D. Zhang, Rev. Sci. Instrum. 85, 093508 (2014)

[9] S. Schmuck, J. Fessey, T. Gerbaud, B. Alper, M.N.A. Beurskens, E. de la Luna, A. Sirinelli, M. Zerbini, JET-EFDA Contributors, Rev. Sci. Instrum. 83, 125101 (2012)

[10] A. Ouroua, J. Beno, A. Bryant, A. Khodak, P. Phillips, W. Rowan, G. Taylor, D. Weeks, IEEE TRANSACTIONS ON PLASMA SCIENCE 46 , 1239 (2018)

[11] D. Farina, L. Figini, P. Platania, C. Sozzi, AIP Conf. Proc. 988, 128 (2008)

[12] T. Zhou, Y. Liu, A. Ti, L. Figini, H. Zhao, Z. Zhu, B. Ling, Fusion Science and Technology 74, 154 (2018) 